\title{
Los libros de autoayuda y la búsqueda de la felicidad
}

DOI: $\underline{\text { https://doi.org/10.32870/dse.v0i11.278 }}$

\section{Teresita Montiel*}

La alta demanda de lecturas de autoayuda especialmente entre los adolescentes puede explicarse tanto por la necesidad de respuestas existenciales que nos permitan entender nuestro sitio en el mundo, como por la incapacidad de encontrar estas respuestas en los marcos institucionales que antes nos quitaban esa incertidumbre.

Uno de los derroteros que nos permitieron encontrar una salida a esta búsqueda de sentido fue por mucho tiempo la religión. Sin embargo, hoy en día existe la tendencia a modificar los patrones de comportamiento religioso, especialmente en los jóvenes. Encuestas europeas dan cuenta de que los jóvenes se consideran creyentes o espirituales pero sin situarse necesariamente en el contexto religioso institucional (EVS, 2011 ${ }^{1}, 2011^{2}$; ISSP Research Group, 2000, 2012). Posiblemente la religiosidad light no permite obtener respuestas a los conflictos que viven los jóvenes día a día.

Por otra parte, la búsqueda de la felicidad en el ser humano, ya ha dejado de ser un simple anhelo individual, actualmente ser feliz se ha convertido en una preocupación que ha derivado en políticas públicas. Países como Japón, Corea del Sur y Brasil han incluido a la felicidad como un derecho constitucional (Lombera, 2012); en el caso de Japón, el artículo 13 de su Constitución (1947) señala que:

Todos los ciudadanos serán respetados como personas individuales. Su derecho a la vida, a la libertad y al logro de la felicidad, será, en tanto que no interfiera con el bienestar público, el objetivo supremo de la legislación y de los demás actos de gobierno.

En el ámbito internacional, en 2011 la Asamblea General de la Organización de las Naciones Unidas determinó medir la felicidad de los habitantes de los países miembros, como un medio para evaluar el progreso social y guiar las políticas públicas. En 2012 se publicó el primer Reporte Mundial de Felicidad, en donde se establecieron los parámetros para medir y explicar el bienestar subjetivo, además de presentar los resultados obtenidos por la Encuesta Mundial de Gallup de 2005 a 2011 en 156 países (Helliwel, Layard y Sachs, 2015).

\footnotetext{
* Doctora en educación, profesora investigadora
} 
¿Pero qué es la felicidad? ¿Es una meta? ¿Es un ideal por el cual luchar? ¿Es un estado del ánimo? De manera general, la psicología ha abordado el tema de la felicidad desde dos miradas, una de ellas tiene que ver con el propósito o sentido de vida y el desarrollo humano, es decir, con un componente eudaimónico, dentro de esta mirada podemos ubicar a los aportes hechos por la psicología humanista y la psicología positiva, quienes suelen utilizar el término de bienestar psicológico.

Por otra parte, hay quienes se refieren al bienestar subjetivo para hablar de la felicidad desde su vertiente hedónica, es decir, considerando la satisfacción de vida, la preponderancia de afectos positivos sobre los negativos, y la evaluación global de la vida (Diener, 1984). Actualmente ambas miradas se consideran complementarias, de manera que permiten ver de una forma más integral el estado de bienestar del ser humano, considerando tanto la emoción como la cognición (Díaz, Stavraki, Blanco y Gandarillas, 2014).

Ahora bien, ¿podemos ser felices a través de la lectura? La respuesta a esta pregunta parece ser algo muy personal; en sociedades alfabetizadas hay quienes disfrutan leer y pueden experimentar estados placenteros a través de un texto, mientras que también hay quienes no creen posible que la lectura pueda traerles cualquier tipo de satisfacción y se consideran a sí mismos como no-lectores (sin caer en cuenta de todos los actos de lectura que realizan de manera cotidiana).

En el ámbito psicoterapéutico las escuelas de psicología derivadas de la corriente conductual, tal como la cognitivo-conductual, ponen el énfasis del tratamiento en llevar a cabo acciones específicas, la mayoría de ellas a realizarse fuera de la consulta, que le ayudan al paciente a generar nuevos hábitos de vida y sistemas de creencias más saludables. En este tipo de enfoque la lectura de un libro puede ser una de las herramientas a utilizar para lograr el cambio en la cognición de la persona.

Basados en esto, se ha desarrollado la biblioterapia como una alternativa al tratamiento psicoterapéutico, donde el paciente recibe un tratamiento estructurado por escrito, ya sea en forma impresa a través de un libro o por medio de una computadora.

¿Quiere decir que los libros de autoayuda pueden sernos útiles en nuestra búsqueda del bienestar emocional?, pues no necesariamente. Los libros utilizados en la biblioterapia no son libros de autoayuda en donde sólo se definan los males que nos aquejan o nos muestren una visión romántica de la vida, sino que se trata de manuales estructurados donde se indican pasos concretos, acciones específicas que permitirán al paciente disminuir su sintomatología con un seguimiento mínimo de parte del terapeuta (Cuijpers, 1997).

Además de libros, también se han utilizado videos y audios como parte de las herramientas consideradas dentro de la terapia psicológica. Tanto la biblioterapia como el uso de medios audiovisuales han probado su efectividad para casos de ansiedad y depresión (Cuijpers, 1997; Hirai y Clum, 2006). Sin embargo, hay que recalcar que la eficacia de estos tratamientos, en donde el paciente es más independiente, descansa precisamente en el diagnóstico y seguimiento que un profesional le proporcione (Febbraro, Clum, Roodman y Wright, 1999). 
Por otra parte, el bienestar emocional es influido por múltiples factores, no sólo depende de la forma en que interpretamos la realidad; por ejemplo, se ha observado que el ingreso del hogar y la expectativa de tener una vida saludable tienen efectos significativos sobre la evaluación de la satisfacción de vida, mientras que el soporte social influye tanto en la satisfacción de vida como en la presencia de emociones positivas (Helliwel et al., 2015).

De manera que todo parece indicar que la lectura de libros de autoayuda es suficiente para encontrar el bienestar emocional anhelado. Ahora bien, ¿la escuela tendría que promover el bienestar emocional? Hoy en día la escuela es prácticamente el segundo hogar de niños y jóvenes, lo cual la convierte en un espacio privilegiado para el desarrollo personal y comunitario. Un estudio realizado por Ashdown y Bernard (2012) con niños pequeños, dio cuenta de que la enseñanza explícita y la práctica de habilidades prosociales y de manejo emocional influyen no sólo en la conducta sino también en el rendimiento académico. ¿Valdría la pena incorporarlo en nuestras aulas?

\section{Referencias}

Ashdown, D. M. y M. E. Bernard (2012). Can Explicit Instruction in Social and Emotional Learning Skills Benefit the Social-Emotional Development, Well-being, and Academic Achievement of Young Children? Early Childhood Education Journal, 39: 397-405.

Diener, E. (1984) Subjecive Well-Being. Psychological Bulletin, 95, 542-575.

EVS (2011) EVS - European Values Study 1981 - Integrated Dataset. GESIS Data Archive, Cologne. ZA4438 Data file Version 3.0.0, doi:10.4232/1.10791

EVS (2011) European Values Study 2008: Integrated Dataset (EVS 2008). GESIS Data Archive, Cologne. ZA4800 Data file Version 3.0.0, doi:10.4232/1.11004

Excélsior (2012). http://www.excelsior.com.mx/2012/02/24/nacional/813314

Cuijpers, P. (1997). Bibliotherapy In Unipolar Depression: A Meta-Analysis. Journal of Behavior Therapy and Experimental Psychiatry, 28, 139-147.

Febbraro, G. A. R., G. A. Clum, A. A. Roodman y J. H. Wright (1999). The Limits of Bibliotherapy: A Study of the Differential Effectiveness of Self-Administered Interventions in Individuals with Panic Attacks. Behavior Therapy, 30: 209-222.

Helliwel, J., R. Layard y J. Sachs (eds.) (2015). World happiness report 2015. Nueva York: Sustainable Development Solutions Network.

Japón (1947). Constitución Política. Recuperado el 1 de septiembre de 2015 de: www.cu.embjapan.go.jp/es/docs/constitucion japon.pdf

Lombera, M. (2012). "Países declaran el derecho a la felicidad". Excélsior, 24 de febrero de 2012. Recuperado el 1 de septiembre de 2015 de: http://www.excelsior.com.mx/2012/02/24/nacio$\underline{\mathrm{nal} / 813314}$ 